Haya: The Saudi Journal of Life Sciences

Abbreviated Key Title: Haya Saudi J Life Sci ISSN 2415-623X (Print) |ISSN 2415-6221 (Online) Scholars Middle East Publishers, Dubai, United Arab Emirates Journal homepage: https://saudijournals.com/sjls

Original Research Article

\title{
Interaction of Urea with Acridinedione Dye-Protein Complex in Aqueous Solution: A Photophysical Approach
}

\author{
Arumugam Dhamodaran ${ }^{1}$, Thamarai Selvan Ramachandran ${ }^{2}$ and Rajendran Kumaran ${ }^{3 *}$ \\ ${ }^{1,2}$ Department of Physical Chemistry, University of Madras, Guindy, Chennai 600 025, Tamil Nadu, India \\ ${ }^{3}$ Department of Chemistry, Dwaraka Doss Goverdhan Doss Vaishnav College (Autonomous) (Affiliated to University of Madras) 833, Gokul Bagh, \\ E.V.R.Periyar Road, Arumbakkam, Chennai 600 106, Tamil Nadu, India
}

DOI: $10.36348 /$ sjls.2020.v05i05.004 $\quad$ | Received: 15.05.2020 | Accepted: 26.05.2020 | Published: 28.05 .2020

*Corresponding author: Rajendran Kumaran

\section{Abstract}

Photophysical studies of water soluble Photoinduced Electron Transfer (PET) based dye with urea in the absence and presence of a globular protein, Bovine Serum Albumin (BSA) were carried out in water. Addition of urea results in a fluorescence quenching of the dye such that the local excited (LE) state emission remains largely unaffected, whereas BSA results in a fluorescence quenching accompanied with larger extent of fluorescence enhancement and promotes the formation of a new emissive peak, assigned to the Charge Transfer (CT) nature of the dye. The loss of PET behavior of the dye in water and the subsequent addition of urea or BSA influences the excited state nature of the dye. Urea predominantly governs the LE state nature of the dye and promotes the formation a new microenvironment in the presence of BSA. The binding constant parameters portray that dye is influenced by urea rather than BSA such that ureawater and urea-urea hydrogen-bonding assemblies predominates over hydrophobic nature of the protein. Fluorescence spectral technique is employed as a tool in establishing the nature of interaction between dyes with more than one water soluble Competitive Hydrogen Bonding solute is established in the present study.

Keywords: Acridinedione dye; Urea; Globular protein; fluorescence emission: binding constant; hydrogen-bonding.

Copyright @ 2020: This is an open-access article distributed under the terms of the Creative Commons Attribution license which permits unrestricted use, distribution, and reproduction in any medium for non-commercial use (NonCommercial, or CC-BY-NC) provided the original author and source are credited.

\section{INTRODUCTION}

The solvation properties and intermolecular interactions of a well-known globular protein like bovine serum albumin, (BSA) with universal protein denaturant, urea in aqueous solution has been well established in the literature by thermodynamical aspects [1-5], photophysical techniques [6-8] and Circular Dichroism (CD) studies [9,10].The hydrogen-bonding properties of urea-water self-assemblies protein -protein interactions in urea aqueous solution and the thermodynamic exchange process of water/urea molecules at the protein solvent interface has provided the chemists and biologists a fascinating field of research. In particular, when the solvent is water, there exists a variation in the equilibrium constant; binding constant and extent of hydrogen-bonding network of urea-protein interaction and this is predominantly attributed for the difference in composition between the bulk solvent and the protein solvation layer. There are reports that authenticate that urea preferentially bind to the protein surface and also reports that include several electrostatic and coulombic interactions that are dependent on the concentration of urea [11-13].
In our present study, we focus on the extent of binding and the nature of interaction of a water soluble extrinsic fluorophore with urea in the absence and presence of BSA. The fluorophore is resorcinol based acridinedione dye (RADD1) that exhibits Photoinduced Electron Transfer (PET) characteristics in non-polar solvents, but loses its PET nature in water and protic solvents (Scheme 1). Both resorcinol and dimedone based acridinedione dyes possess PET and Intramoelcular Charge Transfer (ICT) characteristics such that several photophysical studies pertaining to hydrogen-bonding solutes [14-16] have been carried out in aqueous medium. Herein, we carried out the binding of urea with RADD1 dye in the absence and presence of BSA in water in comparison with another PET dye (RADD2) that exhibits PET behaviour in water and a typical non-PET dye (RADD3). Since the behaviour of urea and BSA with RADD2 and RAAD3 dye has been thoroughly established in water, we have chosen a dye that generally exhibits PET nature in all other solvents except in water and methanol. 


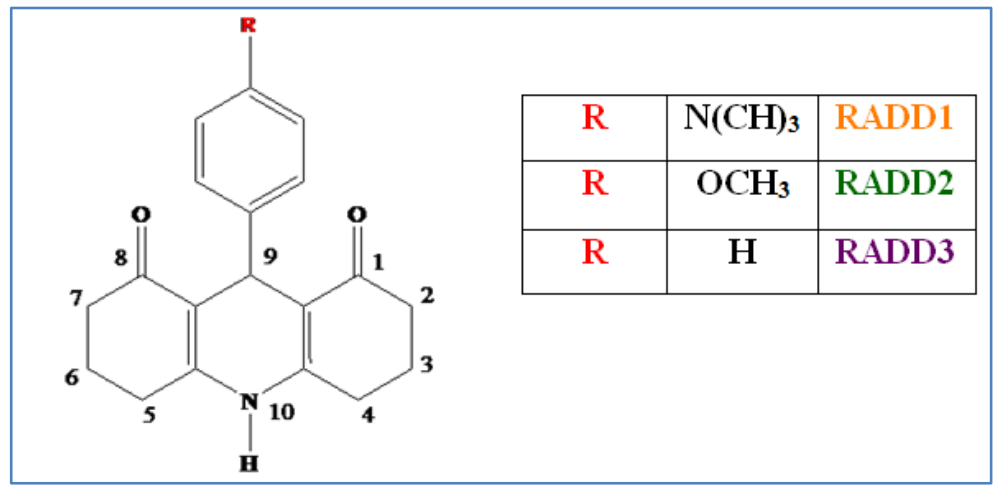

Scheme 1: Structure of Resorcinol based acridinedione dyes

\section{EXPERIMENTAL METHODS Materials}

Resorcinol and dimedone based acridinedione dyes were synthesized as per the procedure reported [17]. Urea (molecular biological grade) was purchased from Merck, India Ltd. BSA fraction V powder $\mathrm{pH}-7$, was purchased from SRL Chemicals India Ltd., and was refrigerated at $4^{\circ} \mathrm{C}$ throughout the course of experimental studies.

\section{Steady-state absorption and fluorescence measurements}

The absorption spectra were recorded using an Agilent 8453 UV-Visible diode array spectrophotometer. The concentration of RADD1 dye was fixed at $3.0 \times 10^{-5} \mathrm{M}$ for all photophysical studies. Emission spectra and Red Edge Excitation Shift (REES) spectral studies were carried out in PerkinElmer MPF-44B fluorescence spectrophotometer interfaced with PC through Rishcom-100 multimeter.

\section{RESULTS AND DISCUSSION}

The absorption spectrum of RADD1 dye in water exhibits maxima around 255 and $375 \mathrm{~nm}$ and the peak at $375 \mathrm{~nm}$ is assigned to the ICT from the nitrogen atom $\left(10^{\text {th }}\right.$ position) to carbonyl group of acridinedione fluorophore [18]. Further, it has been established that the addition of hydrogen-bonding solutes like urea and globular proteins like BSA does not influence the ICT absorption maximum $(375 \mathrm{~nm})$ of the dye and the increase in the absorbance in the spectral range of 250$300 \mathrm{~nm}$ is attributed to the strong absorbance of urea [14] and BSA [19] such that the ICT peak is hardly influenced by the presence of these solutes. Moreover, the increase in the absorbance less than $300 \mathrm{~nm}$ generally does not govern the excited state properties of the dye, since the wavelength of excitation in our present study is around $375 \mathrm{~nm}$ only. Even though some variation in the absorption spectra of ADR results on the addition of urea or BSA, no significant information regarding the ground state characteristics of dye could be established with better information concerned about the nature of inetraction. The precise nature of interaction between urea-dye could not established from the absorption spectra, as observed in the case of urea and protein interaction with other acridinedione dyes in water [14, 19-21].

The emission spectrum recorded by exciting the dye at the longest wavelength absorption maximum is dependent on the electron donating property of the functional groups present at the $9^{\text {th }}$ position of acridinedione dyes and also based on the substitution of hydrogen atom $(\mathrm{N}-\mathrm{H})$ in the $10^{\text {th }}$ position (Scheme 1 ). The unique feature of RADD1 dye is that the PET behaviour is lost in water and other protic solvents such that it exhibits only one emission peak around $438 \pm 2$ $\mathrm{nm}$ in water as shown in figure 1 . In non-protic solvents like acetonitrile, in addition to the LE state, an emission peak above $500 \mathrm{~nm}$ is obtained which corresponds to the CT state [18].

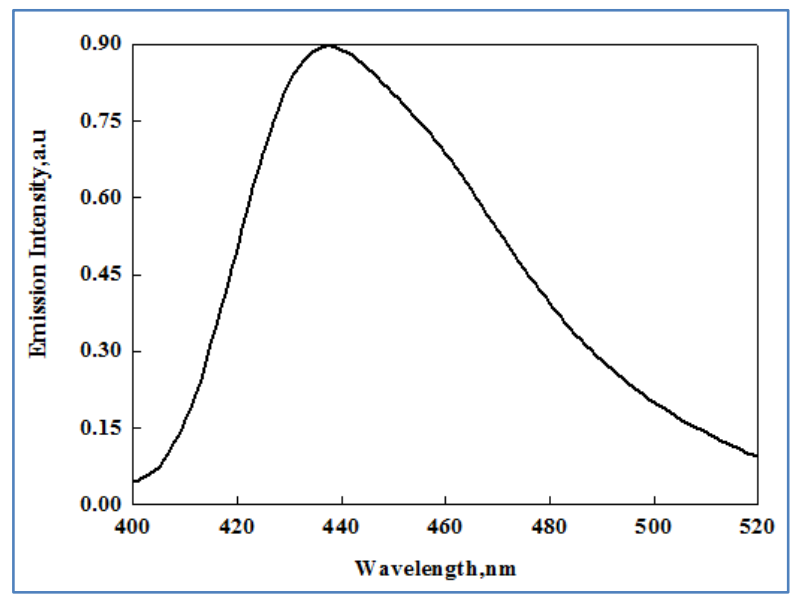

Fig-1: Emission spectra of RADD1 dye in water

This is attributed to the protonation of the dimethylamino group such that the PET character is completely lost and the emission spectral behaviour is entirely different from that in acetonitrile. In protic solvents like water and methanol, protonation of the dimethylamino group increases the oxidation potential of donor group thereby resulting in a large variation in the fluorescence lifetime and the dye thereby losses its PET nature. So far concerned, we have established the photophysical properties of RADD1 and RADD2 dyes with proteins [19,20] and amino acids [22] in aqueous medium and the variation in the excited state properties were found to be entirely different from that of other 
PET and non-PET based acridinedione dyes in water as shown in scheme 1 .

Initial addition of urea (up to $3.0 \mathrm{M}$ ) resulted in a drastic decrease in the fluorescence intensity of ADR dye accompanied with a slight shift in the emission maxima towards the blue region. Further, addition of urea above $3.0 \mathrm{M}$ resulted in an increase in the fluorescence intensity accompanied with a shift towards the red region although the increase in the emission intensity is not comparable to that of the extent in quenching pattern. The fluorescence quenching phenomenon was more predominant, followed by a slight increase in the fluorescence intensity. Although, addition of BSA also resulted in an initial decrease in the fluorescence intensity of the dye, a more pronounced shift and larger fold of enhancement was observed in the case of BSA.

The emission spectra of RADD1dye with urea are shown in figure 2 and the shift in the emission maxima in figure 3 .

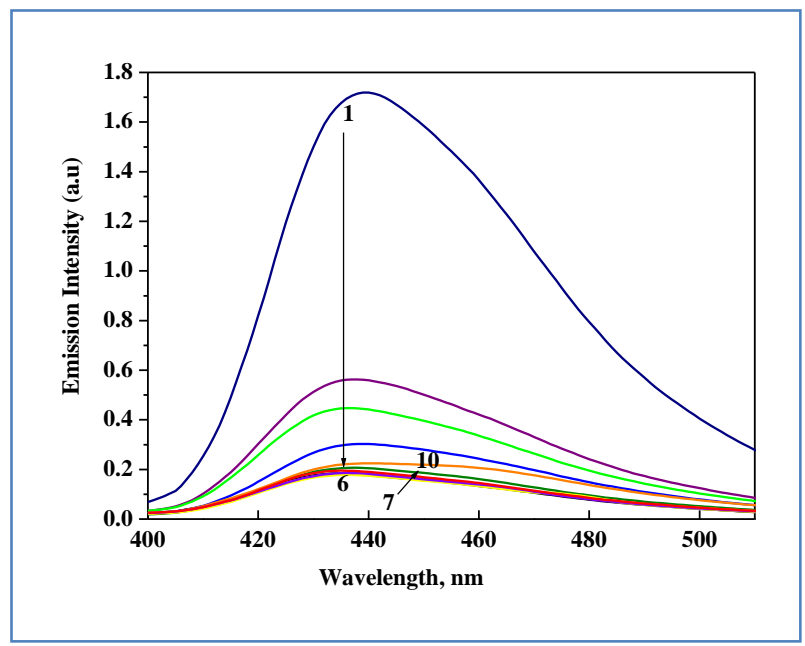

Fig-2: Emission spectra of ADR dye with urea in water

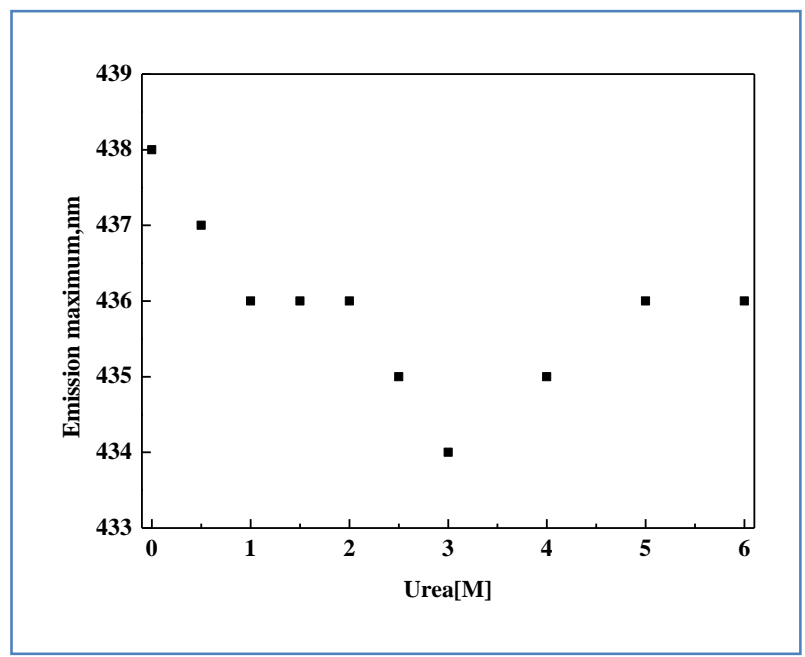

Fig-3: Extent of shift in the emission maxima of RADD1dye dye on the addition of urea.
Interestingly, the initial addition of BSA also quenches the native LE state emission of ADR dye by over $50 \%$ and on the subsequent addition of BSA, a fluorescence enhancement accompanied with a more significant shift towards the red region in the emission maxima (439-470 $\mathrm{nm}$ ) results [20]. This pattern is correlated to the increase in the polarity of the medium and the shift is attributed to the hydrogen-bonding of BSA with dye. In our present study, neither a substantial increase in the fluorescence intensity nor shift in the emission towards the red region results on the addition of urea even though the concentration of urea is several folds greater than that of the protein molecule. In our earlier studies involving BSA with RADD1 dye, the variation in the emission intensity and shift in the emission maxima was more pronounced at a lower concentration of BSA. (Dye: protein was 1:10) and in the case of RADD2 dye, the extent of fluorescence enhancement was very significant accompanied with a blue shift in the mission maxima. Neither of the behavior was observed in the case of addition of urea to RADD1 or RADD2 dye. The nature of the solute and the properties of solute with solvent molecules govern and influence the excited state properties of acridinedione dyes are certainly exemplified in this study, whether the dye is a PET or non-PET based. This clearly ascertains that even though the concentration of urea is around $10^{6}$ fold greater than the dye; the excited state characteristics are influenced to a greater extent. Urea promotes the PET process in RADD1 dye resulting in a fluorescence quenching and suppresses the PET process in the case of RADD2 dye accompanied with an increase in the fluorescence quantum yield. Interestingly, the addition of urea to RADD3 dye does not result any significant change in the fluorescence emission or lifetime of RADD3 dye [14]. Based on the role of urea, we portray that the nature of solute plays an important role on the fluorescence properties, the solute-solvent and solvent mediated properties certainly influences the microenvironment of the dye.

The binding nature of the dye varies with that of the solute molecules like BSA and urea. The SternVolmer plot of RADD1 dye with urea is shown in figure 4 which exhibits a downward curvature. This plot illustrates that the binding pattern is not uniform and exhibits linearity at lower concentration of urea. The binding constant could not be established due to the multiple binding sites due to the presence of large number of urea molecules. A modified plot is provided in the inset of figure 4 which accounts for the fluorescence intensity for quenching alone (Figure 2: plots 1-6 exhibits a decrease in fluorescence intensity). The stern volmer constant (Ksv) constant was obtained from the slope of the linear plot which was found to be 2.4. The bimolecular quenching constant $(\mathrm{kq})$ values were found to be $3.3 \times 10^{8} \mathrm{M}^{-1} \mathrm{~s}^{-1}$. Kq value in the range of $10^{9}$ signifies that the quencher (urea) 
effectively decreases the fluorescence intensity thereby forming an effective binding with RADD1 dye.
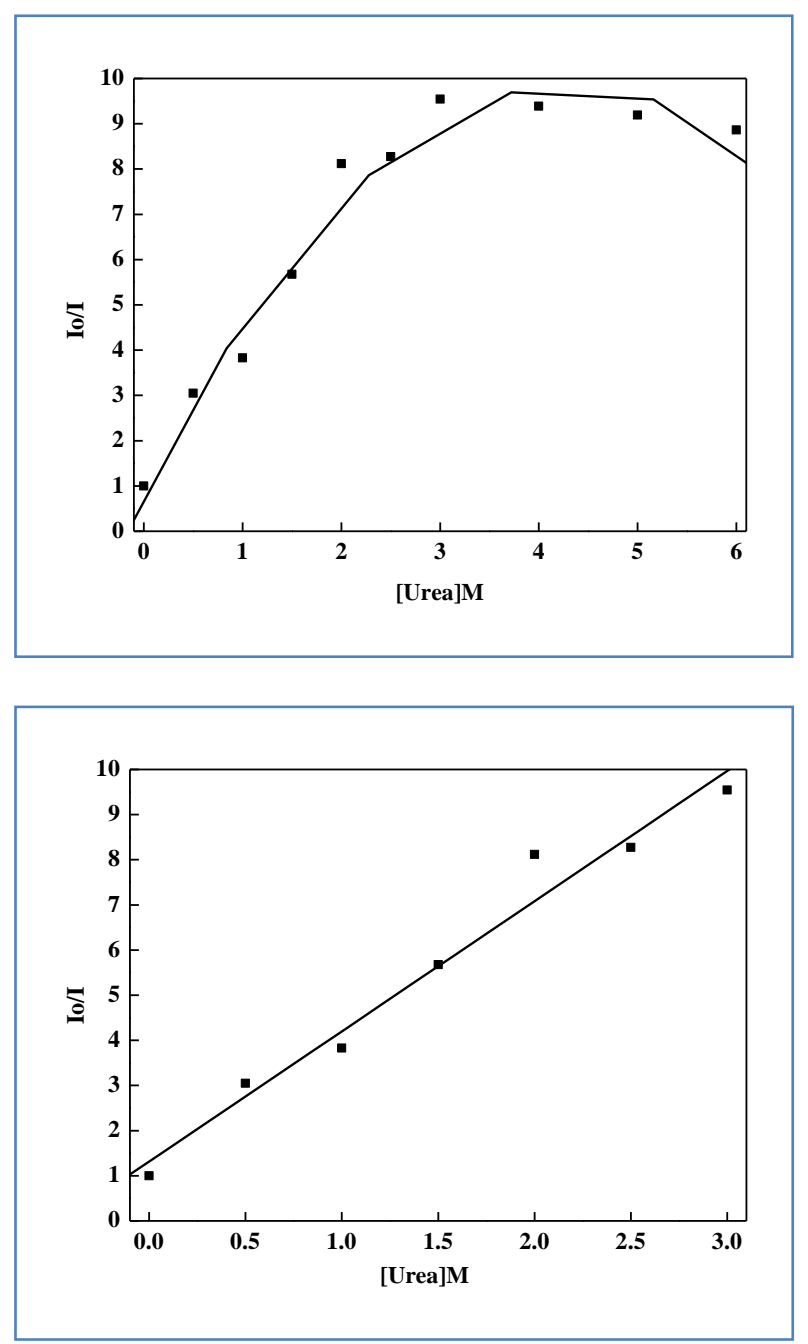

Fig-4: SV plot of RADD1 dye - urea in water

A variation in the binding pattern of dye with urea from that of dye with BSA clearly visualizes that LE state emission is almost retained and do not vary much in the presence of urea. Strikingly, BSA promotes the stabilization of the CT state through hydrogenbonding interaction such that BSA is involved in a direct hydrogen-bonding with RADD1 dye. In the case of urea, there is no clear illustration of any direct hydrogen-bonding with the dye molecule and the ureawater interaction play a major role on the LE state emission of the dye. We portray that more number of urea molecules surround the dye such that it creates a comparatively hydrophobic environment such that the dye switches over towards a change in the microenvironment from hydrophilic to hydrophobic in aqueous medium.

There are several factors involving urea or BSA which induces the LE state emission characteristics such as increase or decrease in the viscosity and dipole moment properties. The red edge effect is an useful and very interesting phenomenon in fluorescence spectroscopy concerned with the emissive nature based on the excitation properties. It is the observation of shifting of emission wavelength to the red upon shifting of the excitation wavelength to the red end of the absorption spectra. The red edge effect is the phenomenon, when we excited a sample at higher wavelength (red edge) the emission maximum shifts to red shift region. Solvation dynamics accompanied with viscous factor is very important for red edge effect and it is observed only in case of highly solvated fluorophores. The role of viscosity of medium is another important factor which is established through Red Edge Excitation Sift (REES) spectral studies. REES spectra was recorded for RADD1 dye alone (absence of urea and BSA), dye with urea and dye-BSA complex with urea. The wavelength range of 373 to 398 $\mathrm{nm}$ with a $5 \mathrm{~nm}$ interval was used for REES studies. Interestingly, the emission maxima centered at $437 \pm 1$ $\mathrm{nm}$ resulted for all the systems in consideration irrespective of the wavelength of excitation.

The REES spectra is provided in figure 5 which clearly authenticates that neither the viscosity factor nor the solvent relaxation dynamics on the fluorophore induced by the addition of urea or BSA has as no significant role on the LE state emission. The presence of urea and urea hydrogen-bonding selfassemblies with water molecules and protein influence the excited state properties is envisaged through this study.

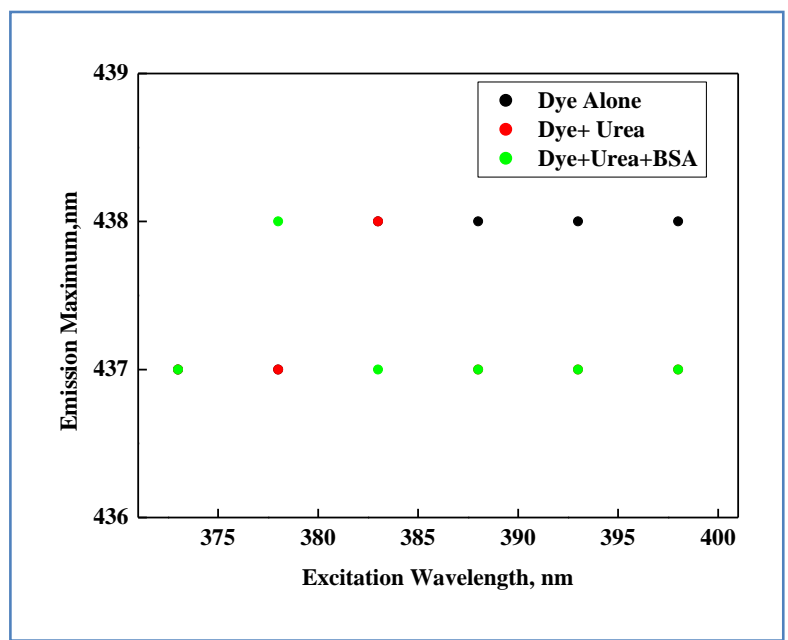

Fig-5: REES spectra of RADD1dye-urea in water in the presence and absence of BSA

Further, we also establish that urea effectively governs the excited state properties of dye in the presence of another competitive solute like BSA wherein BSA influences both the LE and CT state characteristics of the dye. It is well known that BSA forms a stable complex with dye and the binding constant is around $\left(6.3 \times 10^{3} \mathrm{M}^{-1}\right)$ which signifies that the interaction between dye and BSA in aqueous solution is higher in comparison with other CT based dyes[20]. Further, the dye is hydrophobic in nature and prefers to reside inside a hydrophobic cavity rather 
towards a hydrophilic environment. In order to establish the most preferred location of the dye in the presence of competitive solutes, the following emission spectral studies were carried out. The concentration of RADD1 dye-BSA was fixed in the ratio of 1:100 and urea was added to the complex in varying proportion. A decrease in the fluorescence intensity of the dye-BSA complex results and the extent of fluorescence quenching was around 300 fold (figure 6). The concentration of urea was limited to less than $10.0 \mathrm{M}$ in the present study. (Since turbidty at room temperature around $10.0 \mathrm{M}$ in the presence of BSA). The stern volmer plot of urea with dye-BSA complex is provided in figure 7 and the $\mathrm{Ksv}$ and $\mathrm{Kq}$ values were found to be 0.144 and $1.62 \times$ $10^{7} \mathrm{M}^{-1} \mathrm{~s}^{-1}$ respectively.

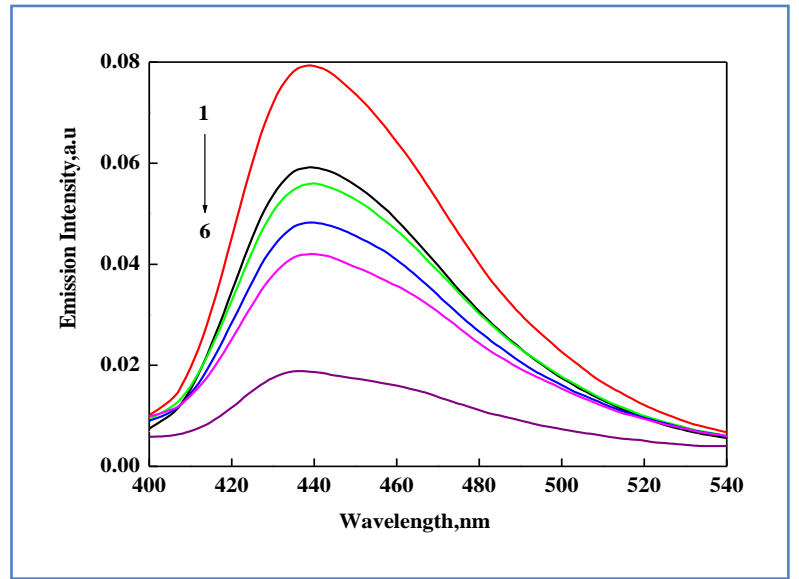

Fig-6: Emission spectra of RADD1 dye-BSA complex with urea

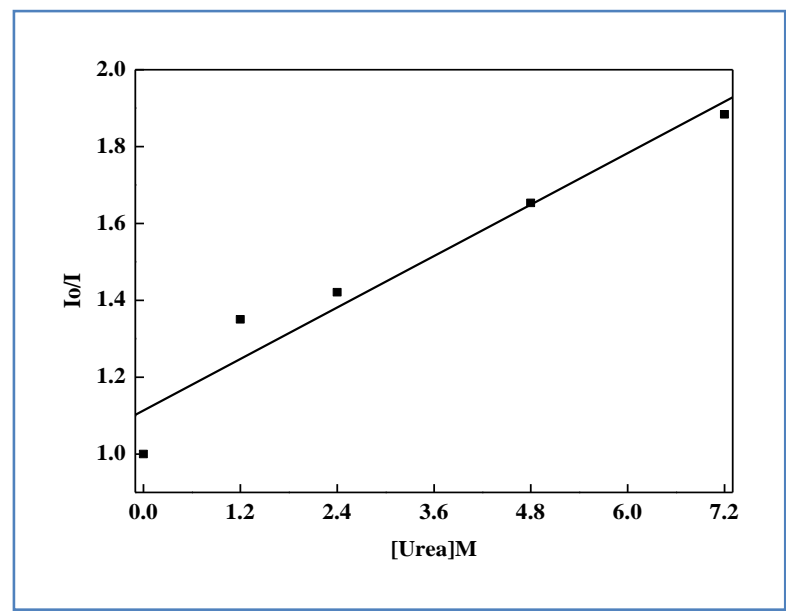

Fig-7: SV plot of RADD1-BSA complex on the addition of urea

From the binding plots, it is evident that urea predominates its role over BSA inspite of a strong dyeBSA Complex. Urea effectively decreases the fluorescence intensity and the dye is oriented more towards a microenvironment containing more urea molecules rather towards BSA. This is authenticated from the binding constant value of urea on dye-BSA complex which is also governed by several entities of urea molecules in comparison with that of BSA. The hydrogen bonding pattern of urea-water does not follow a uniform pattern and based on the concentration of urea there exists various orientation of urea molecules with water forming urea-water, urea-urea and urea-BSA hydrogen bonding assemblies. The presence of urea provides multiple hydrogen-bonding formulations, such that it can act as a hydrogen-bonding donor (N-H hydrogen) as well as an acceptor (carbonyl oxygen). Urea forms hydrogen-bonding with the peptide linkage of protein as well as with the free amino and carboxylate end of the protein molecules thereby resulting in an effective decrease in the binding affinity of BSA with dye. A detailed study on the interaction of urea derivatives with PET based acridinedione dye reveals that apart from hydrogen-bonding interactions, the hydrophobic nature of urea derivatives influences the excited state properties of the dye [14]. Urea creates and promotes the formation of a new micro environment that comprises of hydrogen-bonding and hydrophobic interactions. The stronger urea-urea and urea-water hydrogen-bonding interactions are confined throughout the phase in different proportions such that the microenvironment surrounding dye-BSA complex is completely modified in the presence of urea. We ascertain that in the presence of water as the solvent the role of urea on the protein surface and urea-water selfassemblies influence the excited state properties of the dye

\section{CONCLUSION}

A fluorescence quenching of the dye in the presence of urea reveals that urea diffuses into the fluorophore such that the excited state dynamics are influenced by the presence of urea molecules. The binding constant of dye with urea is unaltered even in the presence of BSA which established that the microenvioernmnet of the dye in the prseence of urea is found to be entirely different from that of BSA. Ureaurea, urea-water hydrogen-bonding self assemblies influnce the fluorescence quantum yeild of the dye is established from fluorescence spectral techniques.

\section{ACKNOWLEDGEMENT}

The authors are thankful to Prof. P. Ramamurthy and Dr. C.Selvaraju of National Centre for Ultrafast Processes, University of Madras, Chennai-600 113, India for permitting us to avail the fluorescence emission and contour facilities. R.K thank Dr. N. Dheenadayalan for the discussions related to the binding studies. The authors are thankful for the support and facilities provided by the Secretary, and Principal, Dwaraka Doss Goverdhan Doss Vaishnav College, Chennai-106. Tamil Nadu, India.

\section{REFERENCES}

1. Sinibaldi, R., Ortore, M. G., Spinozzi, F., de Souza Funari, S., Teixeira, J., \& Mariani, P. (2008). SANS/SAXS study of the BSA solvation properties in aqueous urea solutions via a global fit approach. European Biophysics Journal,37(5), 673-681. 
2. Ortore, M. G., Spinozzi, F., Carsughi, F., Mariani, P., Bonetti, M., \& Onori, G. (2006). High pressure small-angle neutron scattering study of the aggregation state of $\beta$-lactoglobulin in water and in water/ethylene-glycol solutions. Chemical physics letters, 418(4-6), 342-346.

3. Sinibaldi, R., Ortore, M. G., Spinozzi, F., Carsughi, F., Frielinghaus, H., Cinelli, S., ... \& Mariani, P. (2007). Preferential hydration of lysozyme in water/glycerol mixtures: a small-angle neutron scattering study. The Journal of chemical physics, 126(23), 06B609.

4. Spinozzi, F., Mariani, P., Saturni, L., Carsughi, F., Bernstorff, S., Cinelli, S., \& Onori, G. (2007). Metmyoglobin association in dilute solution during pressure-induced denaturation: an analysis at $\mathrm{pH}$ 4.5 by high-pressure small-angle X-ray scattering. The Journal of Physical Chemistry $B, 111(14), 3822-3830$.

5. Zhang, W., Capp, M. W., Bond, J. P., Anderson, C. F., \& Record, M. T. (1996). Thermodynamic characterization of interactions of native bovine serum albumin with highly excluded (glycine betaine) and moderately accumulated (urea) solutes by a novel application of vapor pressure osmometry. Biochemistry, 35(32), 10506-10516.

6. Itri, R., Caetano, W., Barbosa, L. R., \& Baptista, M. S. (2004). Effect of urea on bovine serum albumin in aqueous and reverse micelle environments investigated by small angle X-ray scattering, fluorescence and circular dichroism. Brazilian journal of physics, 34(1), 5863.

7. Kumaran, R., \& Ramamurthy, P. (2011). Denaturation mechanism of BSA by urea derivatives: evidence for hydrogen-bonding mode from fluorescence tools. Journal of fluorescence, 21(4), 1499-1508.

8. Takeda, K., Harada, K., Yamaguchi, K., \& Moriyama, Y. (1994). Conformational changes of bovine serum albumin in an aqueous solution of sodium bis (2-ethylhexyl) sulfosuccinate and in the reverse micelle of the same surfactant. Journal of colloid and interface science, 164(2), 382-386.

9. Lee, S. S., Kiserow, D. J., \& McGown, L. B. (1997). Enzyme solubilization in a reversed micellar microreactor with a bile salt cosurfactant. Journal of colloid and interface science, 193(1), 32-40.

10. Kelly, S. M., \& Price, N. C. (1997). The application of circular dichroism to studies of protein folding and unfolding. Biochimica $E t$ Biophysica Acta-Protein Structure and Molecular Enzymology, 1338(2), 161-185.

11. Timasheff, S. N., \& Xie, G. (2003). Preferential interactions of urea with lysozyme and their linkage to protein denaturation. Biophysical chemistry, 105(2-3), 421-448.
12. Ortore. M.G., Sinibaldi. R., Spinozzi. F. (2009). Sans Study on the Molecular action of denaturants the Lysozyme-urea case, J.R. Soc. Interface, 6, S619-34.

13. Niebuhr, M., \& Koch, M. H. (2005). Effects of urea and trimethylamine-N-oxide (TMAO) on the interactions of lysozyme in solution. Biophysical journal, 89(3), 1978-1983.

14. Kumaran, R., \& Ramamurthy, P. (2006). PET suppression of acridinedione dyes by urea derivatives in water and methanol. The Journal of Physical Chemistry B, 110(47), 23783-23789.

15. Kumaran, R., Varalakshmi, T., Malar, E. P., \& Ramamurthy, P. (2010). Photophysical studies on the interaction of acridinedione dyes with universal protein denaturant: guanidine hydrochloride. Journal of fluorescence, 20(5), 9931002.

16. Kumaran, R., \& Ramamurthy, P. (2011). Photophysical studies on the interaction of formamide and alkyl substituted amides with photoinduced electron transfer (PET) based acridinedione dyes in water. Journal of fluorescence, 21(6), 2165.

17. Srividya, N., Ramamurthy, P., Shanmugasundaram, P., \& Ramakrishnan, V. T. (1996). Synthesis, characterization, and electrochemistry of some acridine-1, 8-dione dyes. The Journal of Organic Chemistry, 61(15), 5083-5089.

18. Thiagarajan, V., Selvaraju, C., Malar, E. P., \& Ramamurthy, P. (2004). A Novel Fluorophore with Dual Fluorescence: Local Excited State and Photoinduced Electron- Transfer- Promoted Charge- Transfer State. ChemPhysChem, 5(8), 1200-1209.

19. Rajendran, K., \& Perumal, R. (2010). Photophysical studies of PET based acridinedione dyes with globular protein: Bovine serum albumin. Journal of luminescence, 130(7), 12031210.

20. Kumaran, R., Vanjinathan, M., \& Ramamurthy, P. (2015). Role of hydrogen-bonding and photoinduced electron transfer (PET) on the interaction of resorcinol based acridinedione dyes with Bovine Serum Albumin (BSA) in water. Journal of Luminescence, 164, 146-153.

21. Sumita, A., Anju, K., Dhenadhayalan, N., Vasanthi, R., \& Kumaran, R. (2020). Competitive hydrogen bonding influences of fluorophore-ureaadenine system in water: Photophysical and photochemical approaches. Spectrochimica Acta Part A: Molecular and Biomolecular Spectroscopy, 118409.

22. Anju, K., Gayathri, S., Sumita, A., Ramamurthy, P., \& Kumaran, R. (2018). Photophysical studies on the interaction of PET and non-PET based acridinedionedyes with glycine in water. Journal of Luminescence, 199, 352-362. 